\title{
Formal Integrability for the Nonautonomous Case of the Inverse Problem of the Calculus of Variations
}

OAna CONSTANTINESCU

Faculty of Mathematics, Alexandru Ioan Cuza University, Bd. Carol no. 11, 700506, Iasi, Romania

E-mail: oanacon@uaic.ro

URL: http://www.math.uaic.ro/ oanacon/

Received March 16, 2012, in final form September 03, 2012; Published online September 06, 2012 http://dx.doi.org/10.3842/SIGMA.2012.059

\begin{abstract}
We address the integrability conditions of the inverse problem of the calculus of variations for time-dependent SODE using the Spencer version of the Cartan-Kähler theorem. We consider a linear partial differential operator $P$ given by the two Helmholtz conditions expressed in terms of semi-basic 1-forms and study its formal integrability. We prove that $P$ is involutive and there is only one obstruction for the formal integrability of this operator. The obstruction is expressed in terms of the curvature tensor $R$ of the induced nonlinear connection. We recover some of the classes of Lagrangian semisprays: flat semisprays, isotropic semisprays and arbitrary semisprays on 2-dimensional manifolds.
\end{abstract}

Key words: formal integrability; partial differential operators; Lagrangian semisprays; Helmholtz conditions

2010 Mathematics Subject Classification: 49N45; 58E30; 34A26; 37J30

\section{Introduction}

One of the most interesting problems of geometric mechanics is related to the integrability conditions of the inverse problem of the calculus of variations for time-dependent second-order ordinary differential equations (SODE). The inverse problem can be formulated as follows. Given a time-dependent system of SODE

$$
\frac{d^{2} x^{i}}{d t^{2}}+2 G^{i}\left(t, x, \frac{d x}{d t}\right)=0, \quad i \in\{1, \ldots, n\},
$$

under what conditions this system can be made equivalent, using a multiplier matrix $g_{i j}$, with the system of Euler-Lagrange equations of a regular Lagrangian

$$
g_{i j}\left(t, x, \frac{d x}{d t}\right)\left(\frac{d^{2} x^{i}}{d t^{2}}+2 G^{i}\left(t, x, \frac{d x}{d t}\right)\right)=\frac{d}{d t}\left(\frac{\partial L}{\partial y^{i}}\right)-\frac{\partial L}{\partial x^{i}} ?
$$

In this case such a system is called variational. The necessary and sufficient conditions under which such a system is variational are known as the Helmholtz conditions.

This inverse problem was solved for the case $n=1$ by Darboux [17], and for $n=2$ by Douglas [20]. Douglas's approach consists in an application of the Riquier theory of systems of partial differential equations [32], to a certain associated linear differential system. The generalization of its results in the higher dimensional case is a very difficult problem because the system provided by the Helmholtz conditions is extremely over-determined. Some of the first studies of the inverse problem in spaces of arbitrary dimension are those of Davis [18] and Kosambi [26]. 
There are different attempts to solve this problem. First, there are some reformulations of the Helmholtz conditions in better geometric forms, which are close enough to the first analytical formulations [20, 33, 34, 35], but undercover more of the geometry behind them [11, 13, 15, 16, 19, 28, 29]. The system of SODE is identified with a semispray on the first jet bundle of a fibred manifold over $\mathbb{R}$. The most important geometric tools induced by a semispray are nonlinear connection, Jacobi endomorphism, dynamical covariant derivative, linear connections and their curvatures. Some reformulations of the Helmholtz conditions are using either the special derivations along the tangent bundle projection introduced in [38], or the semi-basic 1 -forms [5] and the Frölicher-Nijenhuis theory of derivations on the algebra of vector-valued forms [21].

Anderson and Thompson [2] analyzed the inverse problem based on the exterior differential system approach [4]. Using the variational bicomplex associated to a system of arbitrary order ordinary differential equations, they derived the fundamental system of equations for the variational multiplier and proved their sufficiency. They made a detailed study of two dimensional sprays and they proved, for general degrees of freedom, that all isotropic semisprays are variational. It means semisprays that have the associated Jacobi endomorphism a multiple of the identity. This correspond to the Case I of Douglas's classification. This approach is continued in [1], where the case of $\Phi$ diagonalizable, with distinct eigenfunctions, is exposed in detail. The same Case I was proved to be variational also in [36]. This paper uses Riquier theory, but in a more geometric way. The process of repeated differentiations of equations and searching for new nontrivial relations is realized by intrinsic operations.

Another subcase of Douglas's case II is discussed in [14]: separable systems of SODE. Any systems of SODE from this subcase is variational. They showed that any system of SODE in Case II1 with $n$ degrees of freedom can be separated into n separate systems of two firstorder equations. They also proved that there are systems separable in the above sense but not separable into single independent second-order equations. This case was treated in [9]. In [37] the authors reinvestigated the case $n=2$ with their more intrinsic version of the Riquier algorithm. Their approach is based on the same underlying methodology as the analytical work of Douglas.

Another method of studying the integrability conditions of the inverse problem of the calculus of variation is the Spencer-Goldsmchmidt theory of formal integrability of partial differential operators, using two sufficient conditions provided by Cartan-Kähler theorem [12, 22, 40]. This method was applied for autonomous SODE in [23], using the Frölicher-Nijenhuis theory of derivations of vector-valued differential forms. Grifone and Muzsnay gave the first obstructions so that a spray (homogeneous semispray) is variational, for general degrees of freedom. In order to obtain a complete classification of variational sprays, they restricted their work to some particular cases. The Spencer theory is fully applied to the two dimensional case, corresponding to Douglas's paper. For the general n-dimensional case, it is proved only that isotropic sprays are variational. It is important to notice that Grifone and Muzsnay's analysis starts from the Euler-Lagrange partial differential operator, and not from the Helmholtz conditions.

For time independent, homogeneous SODE, the inverse problem is known as the projective metrizability problem. This problem and its formal integrability is studied in [7] using Spencer theory. It was shown that there exists only one first obstruction for the formal integrability of the projective metrizability operator, expressed in terms of the curvature tensor of the nonlinear connection induced by the spray. This obstruction correspond to second obstruction for the formal integrability of the Euler-Lagrange operator.

An interesting and new approach regarding variational PDE's is the one of A. Prástaro $[30,31]$. Using suitable cohomologies and integral bordism groups, the author characterizes variational systems constrained by means of PDE's of submanifolds of fiber bundles. He presents a new algebraic topological characterization of global solutions of variational problems. 
In this paper we address the integrability conditions of the inverse problem of the calculus of variations for time-dependent SODE using also the Spencer version of the Cartan-Kähler theorem. The proper setting is the first jet bundle $J^{1} \pi$ of an $(n+1)$ manifold $M$ fibred over $\mathbb{R}$. In [5] it is proved that a time-dependent semispray is Lagrangian if and only if there exists a semi-basic 1-form $\theta$ on $J^{1} \pi$, that satisfies a differential system. This gives rise to a linear partial differential operator $P$. We study the formal integrability of $P$ using two sufficient conditions provided by Cartan-Kähler theorem. We prove that the symbol $\sigma^{1}(P)$ is involutive (Theorem 3) and hence there is only one obstruction for the formal integrability of the operator $P$, which is due to curvature tensor $R$ (Theorem 4). Based on this result, we recover some of the classes of Lagrangian semisprays: flat semisprays, isotropic semisprays and arbitrary semisprays on 2-dimensional jet spaces $(n=1)$.

The motivation for this article is double-folded. So far all the results about the inverse problem of the calculus of variations were obtained separately, in the autonomous and nonautonomous settings. This is due to the different frameworks involved: the tangent bundle $T M$ (a vector bundle) and respectively the first jet bundle $J^{1} \pi$ (an affine bundle). The geometric tools are usually constructed in different ways, and special attention was given to the timedepending situation. This paper follows the line of [7] but naturally the proofs of the main theorems have some particularities due to the different setting.

Secondly, there are similarities between the formulation of the Helmholtz conditions for sprays in the autonomous setting and respectively for semisprays in the nonautonomous one [5, 6]. This is natural because $J^{1} \pi$ can be embedded in $\widetilde{T M}$ (the tangent bundle with the zero section removed). Due to this embedding one can associate to any regular Lagrangian on the velocityphase space $J^{1} \pi$ a homogeneous degenerate Lagrangian on the extended phase space $\widetilde{T M}$, such that the action defined by a curve in the jet formalism coincides with the action defined by the corresponding curve in the extended formalism. There are correspondences between the main geometric objects associated to these Lagrangians: Poincaré 1- and 2-forms, energies, canonical semisprays-sprays $[3,8,10,24]$. Therefore, due to this homogeneous formalism, it is natural to expect such kind of similarities between the results corresponding to homogeneous structures on $\widetilde{T M}$ and nonhomogeneous one on $J^{1} \pi$.

The paper is organized as follows. In Section 2 we introduce the principal geometric tools induced by a time-dependent semispray on $J^{1} \pi$ and characterize Lagrangian vector fields with respect to semi-basic 1-forms. Section 3 is dedicated to the application of the Spencer theory to the study of formal integrability of the partial differential operator (PDO) $P=\left(d_{J}, d_{h}\right)$. The most important results are Theorems 3 and 4. Section 3.3 presents classes of semisprays for which the obstruction in Theorem 4 is automatically satisfied. For these classes, the PDO $P$ is formally integrable, and hence these semisprays will be Lagrangian SODE.

\section{Preliminaries}

\subsection{The first-order jet bundle $J^{1} \pi$}

The appropriate geometric setting for the study of time-dependent SODE is the affine jet bundle $\left(J^{1} \pi, \pi_{10}, M\right)$ [39]. We consider an $(n+1)$-dimensional, real, smooth manifold $M$, which is fibred over $\mathbb{R}, \pi: M \rightarrow \mathbb{R}$, and represents the space-time. The first jet bundle of $\pi$ is denoted by $\pi_{10}: J^{1} \pi \rightarrow M, \pi_{10}\left(j_{t}^{1} \phi\right)=\phi(t)$, for $\phi$ a local section of $\pi$ and $j_{t}^{1} \phi$ the first-order jet of $\phi$ at $t$. A local coordinate system $\left(t, x^{i}\right)_{i \in\{1, \ldots, n\}}$ on $M$ induces a local coordinate system on $J^{1} \pi$, denoted by $\left(t, x^{i}, y^{i}\right)$. Submersion $\pi_{10}$ induces a natural foliation on $J^{1} \pi$ such that $\left(t, x^{i}\right)$ are transverse coordinates for this foliation, while $\left(y^{i}\right)$ are coordinates for the leaves of the foliation. Throughout the paper we consider Latin indices $i \in\{1, \ldots, n\}$ and Greek indices $\alpha \in\{0, \ldots, n\}$, using the notation $\left(x^{\alpha}\right)=\left(t=x^{0}, x^{i}\right)$. 
In this article we use the Frölicher-Nijenhuis theory [21, 23, 25] of derivations of vector-valued differential forms on the first jet bundle $J^{1} \pi$. We adopt the following notations: $C^{\infty}\left(J^{1} \pi\right)$ for the ring of smooth functions on $J^{1} \pi, \mathfrak{X}\left(J^{1} \pi\right)$ for the $C^{\infty}$ module of vector fields on $J^{1} \pi$ and $\Lambda^{k}\left(J^{1} \pi\right)$ for the $C^{\infty}$ module of $k$-forms on $J^{1} \pi$. The $C^{\infty}$ module of $(r, s)$-type vector fields on $J^{1} \pi$ is denoted by $\mathcal{T}_{s}^{r}\left(J^{1} \pi\right)$ and the tensor algebra on $J^{1} \pi$ is denoted by $\mathcal{T}\left(J^{1} \pi\right)$. The graded algebra of differential forms on $J^{1} \pi$ is written as $\Lambda\left(J^{1} \pi\right)=\bigoplus_{k \in\{1, \ldots, 2 n+1\}} \Lambda^{k}\left(J^{1} \pi\right)$. We denote by $S^{k}\left(J^{1} \pi\right)$ the space of symmetric $(0, k)$ tensors on $J^{1} \pi$ and by $\Psi\left(J^{1} \pi\right)=\bigoplus_{k \in\{1, \ldots, 2 n+1\}} \Psi^{k}\left(J^{1} \pi\right)$ the graded algebra of vector-valued differential forms on $J^{1} \pi$. Throughout the paper we assume that all objects are $C^{\infty}$-smooth where defined.

A parametrized curve on $M$ is a section of $\pi: \gamma: \mathbb{R} \rightarrow M, \gamma(t)=\left(t, x^{i}(t)\right)$. Its first-order jet prolongation $J^{1} \gamma: t \in \mathbb{R} \rightarrow J^{1} \gamma(t)=\left(t, x^{i}(t), d x^{i} / d t\right) \in J^{1} \pi$ is a section of the fibration $\pi_{1}:=\pi \circ \pi_{10}: J^{1} \pi \rightarrow \mathbb{R}$.

Let $V J^{1} \pi$ be the vertical subbundle of $T J^{1} \pi, V J^{1} \pi=\left\{\xi \in T J^{1} \pi, D \pi_{10}(\xi)=0\right\} \subset T J^{1} \pi$. The fibers $V_{u} J^{1} \pi=\operatorname{Ker} D_{u} \pi_{10}, u \in J^{1} \pi$ determine a regular, $n$-dimensional, integrable vertical distribution. Remark that $V_{u} J^{1} \pi=\operatorname{spann}\left\{\partial / \partial y^{i}\right\}$ and its annihilators are the contact 1-forms $\delta x^{i}=d x^{i}-y^{i} d t, i \in\{1, \ldots, n\}$ and basic 1 -forms $\lambda d t, \lambda \in C^{\infty}\left(J^{1} \pi\right)$. The vertical endomorphism $J=\frac{\partial}{\partial y^{i}} \otimes \delta x^{i}$ is a vector-valued 1-form on $J^{1} \pi$, with $\operatorname{Im} J=V\left(J^{1} \pi\right), V\left(J^{1} \pi\right) \subset \operatorname{Ker} J$ and $J^{2}=0$.

Its Frölicher-Nijenhuis tensor is given by

$$
N_{J}=\frac{1}{2}[J, J]=-\frac{\partial}{\partial y^{i}} \otimes \delta x^{i} \wedge d t=-J \wedge d t .
$$

Consequently, $d_{J}^{2}=d_{N_{J}}=-d_{J \wedge d t} \neq 0$ and therefore $d_{J^{-}}$exact forms on $J^{1} \pi$ may not be $d_{J^{-}}$ closed. Here $d_{J}$ is the exterior derivative with respect to the vertical endomorphisms.

Remark 1. For $A \in \Psi^{1}\left(J^{1} \pi\right)$ a vector-valued 1-form, the exterior derivative with respect to $A$ is a derivation of degree 1 given by $d_{A}=i_{A} \circ d-d \circ i_{A}$.

A $k$-form $\omega$ on $J^{1} \pi, k \geq 1$, is called semi-basic if it vanishes whenever one of the arguments is vertical.

A vector-valued $k$-form $A$ on $J^{1} \pi$ is called semi-basic if it takes values in the vertical bundle and it vanishes whenever one of the arguments is vertical.

A semi-basic $k$-form satisfies the relation $i_{J} \theta=0$ and locally can be expressed as $\theta=$ $\theta_{0} d t+\theta_{i} \delta x^{i}$. For example, contact 1-forms $\delta x^{i}$ are semi-basic 1-forms.

If a vector-valued $k$-form $A$ is semi-basic, then $J \circ A=0$ and $i_{J} A=0$. The vertical endomorphism $J$ is a vector-valued, semi-basic 1 -form.

Locally, a semi-basic $k$-form $\theta$ has the next form

$$
\theta=\frac{1}{k !} \theta_{i_{1} \ldots i_{k}}\left(x^{\alpha}, y^{j}\right) \delta x^{i_{1}} \wedge \cdots \wedge \delta x^{i_{k}}+\frac{1}{(k-1) !} \widetilde{\theta}_{i_{1} \ldots i_{k-1}}\left(x^{\alpha}, y^{j}\right) \delta x^{i_{1}} \wedge \cdots \wedge \delta x^{i_{k-1}} \wedge d t .
$$

For simplicity, we denote by $T^{*}$ the vector bundle of 1 -forms on $J^{1} \pi$, by $T_{v}^{*}$ the vector bundle of semi-basic 1-forms on $J^{1} \pi$ and by $\Lambda^{k} T_{v}^{*}$ the vector bundle of semi-basic $k$-forms on $J^{1} \pi$. We also denote by $\Lambda_{v}^{k}=\operatorname{Sec}\left(\Lambda^{k} T_{v}^{*}\right)$ the $C^{\infty}\left(J^{1} \pi\right)$-module of sections of $\Lambda^{k} T_{v}^{*}$ and by $S^{k} T^{*}$ the vector bundle of symmetric tensors of $(0, k)$-type on $J^{1} \pi$. $S^{1} T^{*}$ will be identified with $T^{*}$.

A semispray is a globally defined vector field $S$ on $J^{1} \pi$ such that

$$
J(S)=0 \quad \text { and } \quad d t(S)=1 .
$$

The integral curves of a semispray are first-order jet prolongations of sections of $\pi \circ \pi_{10}: J^{1} \pi \rightarrow \mathbb{R}$. Locally, a semispray has the form

$$
S=\frac{\partial}{\partial t}+y^{i} \frac{\partial}{\partial x^{i}}-2 G^{i}\left(x^{\alpha}, y^{j}\right) \frac{\partial}{\partial y^{i}},
$$

where functions $G^{i}$, called the semispray coefficients, are locally defined on $J^{1} \pi$. 
A parametrized curve $\gamma: I \rightarrow M$ is a geodesic of $S$ if $S \circ J^{1} \gamma=\frac{d}{d t}\left(J^{1} \gamma\right)$.

In local coordinates, $\gamma(t)=\left(t, x^{i}(t)\right)$ is a geodesic of the semispray $S$ given by (1) if and only if it satisfies the system of SODE

$$
\frac{d^{2} x^{i}}{d t^{2}}+2 G^{i}\left(t, x, \frac{d x}{d t}\right)=0
$$

Therefore such a system of time-dependent SODE can be identified with a semispray on $J^{1} \pi$.

Canonical nonlinear connection. A nonlinear connection on $J^{1} \pi$ is an $(n+1)$-dimensional distribution $H: u \in J^{1} \pi \mapsto H_{u} \subset T_{u} J^{1} \pi$, supplementary to $V J^{1} \pi: \forall u \in J^{1} \pi, T_{u} J^{1} \pi=H_{u} \oplus V_{u}$.

A semispray $S$ induces a nonlinear connection on $J^{1} \pi$, given by the almost product structure $\Gamma=-\mathcal{L}_{S} J+S \otimes d t, \Gamma^{2}=\mathrm{Id}$. The horizontal projector that corresponds to this almost product structure is $h=\frac{1}{2}\left(\mathrm{Id}-\mathcal{L}_{S} J+S \otimes d t\right)$ and the vertical projector is $v=\operatorname{Id}-h$.

The horizontal subspace is spanned by $S$ and by $\frac{\delta}{\delta x^{i}}:=\frac{\partial}{\partial x^{i}}-N_{i}^{j} \frac{\partial}{\partial y^{j}}$, where $N_{j}^{i}=\frac{\partial G^{i}}{\partial y^{j}}$. In this paper we prefer to work with the following adapted basis and cobasis:

$$
\left\{S, \frac{\delta}{\delta x^{i}}, \frac{\partial}{\partial y^{i}}\right\}, \quad\left\{d t, \delta x^{i}, \delta y^{i}\right\}
$$

with $\delta x^{i}$ the contact 1-forms and $\delta y^{i}=d y^{i}+N_{\alpha}^{i} d x^{\alpha}, N_{0}^{i}=2 G^{i}-N_{j}^{i} y^{j}$. Functions $N_{j}^{i}$ and $N_{0}^{i}$ are the coefficients of the nonlinear connection induced by the semispray $S$.

With respect to basis and cobasis (3), the horizontal and vertical projectors are locally expressed as $h=S \otimes d t+\frac{\delta}{\delta x^{i}} \otimes \delta x^{i}, v=\frac{\partial}{\partial y^{i}} \otimes \delta y^{i}$. We consider the (1,1)-type tensor field $\mathbb{F}=h \circ \mathcal{L}_{S} h-J$, which corresponds to the almost complex structure in the autonomous case. It satisfies $\mathbb{F}^{3}+\mathbb{F}=0$, which means that it is an $f(3,1)$ structure. It can be expressed locally as $\mathbb{F}=\frac{\delta}{\delta x^{i}} \otimes \delta y^{i}-\frac{\partial}{\partial y^{j}} \otimes \delta x^{i}$.

Curvature. The following properties for the torsion and curvature of the nonlinear connection induced by the semispray are proved in [5].

The weak torsion tensor field of the nonlinear connection $\Gamma$ vanishes: $[J, h]=0$, which is equivalent also with $[J, \Gamma]=0$.

The curvature tensor $R=N_{h}$ of the nonlinear connection $\Gamma$ is a vector-valued semi-basic 2-form, locally given by

$$
R=\frac{1}{2}[h, h]=\frac{1}{2} R_{i j}^{k} \frac{\partial}{\partial y^{k}} \otimes \delta x^{i} \wedge \delta x^{j}+R_{i}^{j} \frac{\partial}{\partial y^{j}} \otimes d t \wedge \delta x^{i},
$$

where

$$
R_{j k}^{i}=\frac{\delta N_{j}^{i}}{\delta x^{k}}-\frac{\delta N_{k}^{i}}{\delta x^{j}}
$$

and

$$
R_{j}^{i}=2 \frac{\partial G^{i}}{\partial x^{j}}-\frac{\partial G^{i}}{\partial y^{k}} \frac{\partial G^{k}}{\partial y^{j}}-S\left(\frac{\partial G^{i}}{\partial y^{j}}\right) .
$$

The Jacobi endomorphism is defined as

$$
\Phi=v \circ \mathcal{L}_{S} h=\mathcal{L}_{S} h-\mathbb{F}-J .
$$

Jacobi endomorphism $\Phi$ is a semi-basic, vector-valued 1-form and satisfies $\Phi^{2}=0$. Locally, can be expressed as $\Phi=R_{i}^{j} \frac{\partial}{\partial y^{j}} \otimes \delta x^{i}$, where $R_{j}^{i}$ are given by (5).

The Jacobi endomorphism and the curvature of the nonlinear connection are related by the following formulae:

$$
\begin{aligned}
& \Phi=i_{S} R, \\
& {[J, \Phi]=3 R+\Phi \wedge d t .}
\end{aligned}
$$

Remark that $R=0$ if and only if $\Phi=0$. 
Definition 1. A semispray $S$ is called isotropic if its Jacobi endomorphism has the form

$$
\Phi=\lambda J
$$

where $\lambda \in C^{\infty}\left(J^{1} \pi\right)$.

Next we express the isotropy condition (9) for a semispray in terms of the curvature tensor $R$.

Proposition 1. A semispray $S$ is isotropic if and only if its curvature tensor $R$ has the form

$$
R=\alpha \wedge J
$$

where $\alpha$ is a semi-basic 1 -form on $J^{1} \pi$.

Proof. Suppose that $S$ is an isotropic SODE. Then there exists $\lambda \in C^{\infty}\left(J^{1} \pi\right)$ such that $\Phi=\lambda J$. From (8) it results

$$
\begin{aligned}
& 3 R=[J, \lambda J]-\Phi \wedge d t \\
& {[J, \lambda J]=\left(d_{J} \lambda\right) \wedge J-d \lambda \wedge J^{2}+\lambda[J, J] \Rightarrow R=\frac{1}{3}\left(d_{J} \lambda\right) \wedge J-\lambda J \wedge d t=\alpha \wedge J}
\end{aligned}
$$

with $\alpha=\frac{1}{3} d_{J} \lambda+\lambda d t \in T_{v}^{*}$.

In the above calculus we used the formula [23]

$$
[K, g L]=\left(d_{K} g\right) \wedge L-d g \wedge K L+g[K, L]
$$

for $K, L$ vector-valued one-forms on $J^{1} \pi$ and $g \in C^{\infty}\left(J^{1} \pi\right)$.

For the converse, suppose that $R=\alpha \wedge J$, with $\alpha \in T_{v}^{*}$. Formula (7) implies $\Phi=i_{S}(\alpha \wedge J)=$ $\left(i_{S} \alpha\right) J-\alpha \wedge i_{S} J=\left(i_{S} \alpha\right) J$

\section{$2.2 \quad$ Lagrangian semisprays}

In this subsection we recall some basic notions about Lagrangian semisprays.

\section{Definition 2.}

1) A smooth function $L \in C^{\infty}\left(J^{1} \pi\right)$ is called a Lagrangian function.

2) The Lagrangian $L$ is regular if the $(0,2)$ type tensor with local components

$$
g_{i j}\left(x^{\alpha}, y^{k}\right)=\frac{\partial^{2} L}{\partial y^{i} \partial y^{j}}
$$

has rank $n$ on $J^{1} \pi$. The tensor $g=g_{i j} \delta x^{i} \otimes \delta x^{j}$ is called the metric tensor of the Lagrangian $L$.

Remark 2. More exactly, [39], a function $L \in C^{\infty}\left(J^{1} \pi\right)$ is called a Lagrangian density on $\pi$. If $\Omega$ is a volume form on $\mathbb{R}$, the corresponding Lagrangian is the semi-basic 1-form $L \pi_{1}^{*} \Omega$ on $J^{1} \pi$. Using a fixed volume form on $\mathbb{R}$, for example $d t$, it is natural to consider the function $L$ as a (first-order) Lagrangian.

For the particular choice of $d t$ as volume form on $\mathbb{R}$, the Poincaré-Cartan 1-form of the Lagrangian $L$ is $\theta_{L}:=L d t+d_{J} L$. The Lagrangian $L$ is regular if and only if the PoincaréCartan 2-form $d \theta_{L}$ has maximal rank $2 n$ on $J^{1} \pi$.

For a detailed exposition on the regularity conditions for Lagrangians see [27]. 
The geodesics of a semispray $S$, given by the system of SODE (2), coincide with the solutions of the Euler-Lagrange equations

$$
\frac{d}{d t}\left(\frac{\partial L}{\partial y^{i}}\right)-\frac{\partial L}{\partial x^{i}}=0
$$

if and only if

$$
g_{i j}\left(t, x, \frac{d x}{d t}\right)\left(\frac{d^{2} x^{i}}{d t^{2}}+2 G^{i}\left(t, x, \frac{d x}{d t}\right)\right)=\frac{d}{d t}\left(\frac{\partial L}{\partial y^{i}}\right)-\frac{\partial L}{\partial x^{i}} .
$$

Therefore, for a semispray $S$, there exists a Lagrangian function $L$ such that (10) holds true if and only if $S\left(\frac{\partial L}{\partial y^{i}}\right)-\frac{\partial L}{\partial x^{i}}=0$, which can be further expressed as

$$
\mathcal{L}_{S} \theta_{L}=d L \Leftrightarrow i_{S} d \theta_{L}=0
$$

Definition 3. A semispray $S$ is called a Lagrangian semispray (or a Lagrangian vector field) if there exists a Lagrangian function $L$, locally defined on $J^{1} \pi$, that satisfies (11).

In [5] it has been shown that a semispray $S$ is a Lagrangian semispray if and only if there exists a semi-basic 1 -form $\theta \in \Lambda_{v}^{1}$ with $\operatorname{rank}(d \theta)=2 n$ on $J^{1} \pi$, such that $\mathcal{L}_{S} \theta$ is closed. This represents a reformulation, in terms of semi basic 1-forms, of the result in terms of 2 -forms obtained by Crampin et al. in [15]. The characterization of Lagrangian higher order semisprays in terms of a closed 2-form appears also in [2].

Based on this result we can obtain the following reformulation in terms of semi-basic 1-forms of the known Helmholtz conditions [5, Lemma 4.2, Lemma 4.3, Theorem 4.5, Theorem 5.1].

Theorem 1. A semispray $S$ is a Lagrangian vector field if and only if there exists a semi-basic 1 -form $\theta \in \Lambda_{v}^{1}$, with $\operatorname{rank}(d \theta)=2 n$ on $J^{1} \pi$, such that

$$
d_{J} \theta=0, \quad d_{h} \theta=0 .
$$

Proof. In order to make this paper self contained, we give a direct proof of this theorem.

Suppose that $S$ is a Lagrangian semispray. It results that there exists a regular Lagrangian $L$ on $J^{1} \pi$ with $\mathcal{L}_{S} \theta_{L}=d L$, or equivalently $i_{S} d \theta_{L}=0$, where $\theta_{L}=L d t+d_{J} L$ is its Poincaré 1-form. Evidently $\theta_{L}$ is a semi-basic 1-form with $\operatorname{rank}\left(d \theta_{L}\right)=2 n$ on $J^{1} \pi$. We will prove that $d_{J} \theta_{L}=d_{h} \theta_{L}=0$.

Indeed, $d_{J} \theta_{L}=d_{J} L \wedge d t+L d_{J} d t+d_{J}^{2} L=i_{J} d L \wedge d t-d_{J \wedge d t} L=i_{J} d L \wedge d t-i_{J \wedge d t} d L=0$.

From the formula $i_{J} \mathcal{L}_{S}-\mathcal{L}_{S} i_{J}=i_{\Gamma-S \otimes d t}$ and $i_{J} \mathcal{L}_{S} d \theta_{L}=0$ we obtain $\mathcal{L}_{S} d_{J} \theta_{L}+i_{\Gamma} d \theta_{L}-$ $i_{S \otimes d t} d \theta_{L}=0$. We also compute $i_{\Gamma} d \theta_{L}=i_{2 h-\mathrm{Id}} d \theta_{L}=2 i_{h} d \theta_{L}-2 d \theta_{L}=2 d_{h} \theta_{L}$. Therefore $2 d_{h} \theta_{L}=i_{S \otimes d t} d \theta_{L}=-i_{S} d \theta_{L} \wedge d t=0$.

Conversely, suppose that there exists a semi-basic 1-form $\theta \in \Lambda_{v}^{1}$, with $\operatorname{rank}(d \theta)=2 n$ on $J^{1} \pi$, such that $d_{J} \theta=0, d_{h} \theta=0$. In order to prove that $S$ is a Lagrangian vector field, we will first show that $\mathcal{L}_{S} \theta=d\left(i_{S} \theta\right)$.

The hypothesis $d_{J} \theta=0$ implies $\theta=\left(i_{S} \theta\right) d t+d_{J}\left(i_{S} \theta\right)$. Indeed, $d_{J} i_{S}+i_{S} d_{J}=\mathcal{L}_{J S}-i_{[S, J]}=$ $i_{h-S \otimes d t-v} \Rightarrow d_{J}\left(i_{S} \theta\right)=i_{h} \theta-\left(i_{S} \theta\right) d t-i_{v} \theta=\theta-\left(i_{S} \theta\right) d t$.

Next, from $d_{h} i_{S}+i_{S} d_{h}=\mathcal{L}_{h S}-i_{[S, h]}$ and $d_{h} \theta=0$ it results that $d_{h} i_{S} \theta=\mathcal{L}_{S} \theta-i_{\mathbb{F}+J+\Phi} \theta=$ $\mathcal{L}_{S} \theta-i_{\mathbb{F}} \theta$.

From $i_{\mathbb{F}} \theta=i_{\mathbb{F}}\left(\left(i_{S} \theta\right) d t+d_{J}\left(i_{S} \theta\right)\right)=i_{\mathbb{F}}\left(d_{J}\left(i_{S} \theta\right)\right)$ and $i_{\mathbb{F}} d_{J}-d_{J} i_{\mathbb{F}}=d_{J \circ \mathbb{F}}-i_{[\mathbb{F}, J]} \Rightarrow$ $i_{\mathbb{F}}\left(d_{J}\left(i_{S} \theta\right)\right)=d_{v}\left(i_{S} \theta\right)$. It results that $\mathcal{L}_{S} \theta=d_{h}\left(i_{S} \theta\right)+d_{v}\left(i_{S} \theta\right)=d\left(i_{S} \theta\right)$.

Consider $L=i_{S} \theta$. Then $\theta$ is the Poincaré-Cartan 1 -form of $L$ and $\mathcal{L}_{S} \theta_{L}=d L$. From $\operatorname{rank}(d \theta)=2 n$ on $J^{1} \pi$ it results that $L$ is a regular Lagrangian and $S$ is a Lagrangian vector field.

In the next section we discuss the formal integrability of these Helmholtz conditions using two sufficient conditions provided by Cartan-Kähler theorem. 


\section{Formal integrability for the nonautonomus inverse problem of the calculus of variations}

In order to study the integrability conditions of the set of differential equations (12), we associate to it a linear partial differential operator and study its formal integrability, using Spencer's technique. The approach in this work follows the one developed in [7] for studying the projective metrizability problem for autonomous sprays. For the basic notions of formal integrability theory of linear partial differential operators see [7, 23].

Consider $T_{v}^{*}$ the vector bundle of semi-basic 1-forms on $J^{1} \pi$ and $\Lambda_{v}^{1}$ the module of sections of $T_{v}^{*}$. For $\theta \in \Lambda_{v}^{1}$ and $k \geq 1$ we denote by $j_{u}^{k} \theta$ the $k$ th order jet of $\theta$ at the base point $u$ in $J^{1} \pi$. The bundle of $k$ th order jets of sections of $T_{v}^{*}$ is denoted by $J^{k} T_{v}^{*}$. The projection $\pi_{0}: J^{k} T_{v}^{*} \rightarrow J^{1} \pi$ is defined by $\pi_{0}\left(j_{u}^{k} \theta\right)=u$. If $l>k$, one defines the projections $\pi_{k}$ as follows: $\pi_{k}\left(j_{u}^{l} \theta\right)=j_{u}^{k} \theta$ and $J^{l} T_{v}^{*}$ is also a fibred manifold over $J^{k} T_{v}^{*}$.

If $f_{1}, \ldots, f_{k} \in C^{\infty}\left(J^{1} \pi\right)$ are functions vanishing at $u \in J^{1} \pi$ and $\theta \in \Lambda_{v}^{1}$, we define $\epsilon$ : $\mathcal{S}^{k} T^{*} \otimes T_{v}^{*} \longrightarrow J^{k} T_{v}^{*}$ by $\epsilon\left(d f_{1} \odot \cdots \odot d f_{k} \otimes \theta\right)_{u}=j_{u}^{k}\left(f_{1} \cdots f_{k} \theta\right)$, where $\odot$ is the symmetric product. Then the sequence

$$
0 \longrightarrow \mathcal{S}^{k} T^{*} \otimes T_{v}^{*} \stackrel{\epsilon}{\longrightarrow} J^{k} T_{v}^{*} \stackrel{\pi_{k-1}}{\longrightarrow} J^{k-1} T_{v}^{*} \longrightarrow 0
$$

is exact.

Consider the linear partial differential operator of order one

$$
P: \Lambda_{v}^{1} \rightarrow \Lambda_{v}^{2} \oplus \Lambda_{v}^{2}, \quad P=\left(d_{J}, d_{h}\right) .
$$

Remark that $P(\theta)$ can be expressed in terms of first-order jets of $\theta$, for any $\theta \in \Lambda_{v}^{1}$, and therefore it induces a morphism between vector bundles:

$$
p^{0}(P): J^{1} T_{v}^{*} \rightarrow \Lambda^{2} T_{v}^{*} \oplus \Lambda^{2} T_{v}^{*}, \quad p^{0}(P)\left(j_{u}^{1} \theta\right)=P(\theta)_{u}, \quad \forall \theta \in \Lambda_{v}^{1} .
$$

We also consider the $l$ th order jet prolongations of the differential operator $P, l \geq 1$, which will be identified with the morphisms of vector bundles over $M$,

$$
p^{l}(P): J^{l+1} T_{v}^{*} \rightarrow J^{l}\left(\Lambda^{2} T_{v}^{*} \oplus \Lambda^{2} T_{v}^{*}\right), \quad p^{l}(P)\left(j_{u}^{l+1} \theta\right)=j_{u}^{l}(P(\theta)), \quad \forall \theta \in \Lambda_{v}^{1} .
$$

Remark that for a semi-basic 1-form $\theta=\theta_{\alpha} \delta x^{\alpha}$, its first-order jet $j^{1} \theta=\frac{\delta \theta_{\alpha}}{\delta x^{\beta}} \delta x^{\beta} \otimes \delta x^{\alpha}+$ $\frac{\partial \theta_{\alpha}}{\partial y^{i}} \delta y^{i} \otimes \delta x^{\alpha}$ determines the local coordinates $\left(x^{\alpha}, y^{i}, \theta_{\alpha}, \theta_{\alpha \beta}, \theta_{\alpha \underline{i}}\right)$ on $J^{1} T_{v}^{*}$. In this work all contravariant or covariant indices, related to vertical components of tensor fields will be underlined.

Consider $\theta=\theta_{\alpha} \delta x^{\alpha}$, a semi-basic 1-form on $J^{1} \pi$. Then

$$
\begin{aligned}
d \theta= & \left(\frac{\partial \theta_{i}}{\partial t}-\theta_{j} N_{i}^{j}-\frac{\delta \theta_{0}}{\delta x^{i}}\right) \delta x^{0} \wedge \delta x^{i}+\left(\theta_{i}-\frac{\partial \theta_{0}}{\partial y^{i}}\right) \delta x^{0} \wedge \delta y^{i} \\
& +\frac{1}{2}\left(\frac{\delta \theta_{j}}{\delta x^{i}}-\frac{\delta \theta_{i}}{\delta x^{j}}\right) \delta x^{i} \wedge \delta x^{j}+\left(\frac{\partial \theta_{j}}{\partial y^{i}}\right) \delta y^{i} \wedge \delta x^{j}, \\
d_{J} \theta= & \left(\theta_{i}-\frac{\partial \theta_{0}}{\partial y^{i}}\right) \delta x^{0} \wedge \delta y^{i}+\frac{1}{2}\left(\frac{\partial \theta_{i}}{\partial y^{j}}-\frac{\partial \theta_{j}}{\partial y^{i}}\right) \delta x^{j} \wedge \delta x^{i}, \\
d_{h} \theta= & \left(\frac{\partial \theta_{i}}{\partial t}-\theta_{j} N_{i}^{j}-\frac{\delta \theta_{0}}{\delta x^{i}}\right) \delta x^{0} \wedge \delta x^{i}+\frac{1}{2}\left(\frac{\delta \theta_{i}}{\delta x^{j}}-\frac{\delta \theta_{j}}{\delta x^{i}}\right) \delta x^{j} \wedge \delta x^{i} .
\end{aligned}
$$

Using these formulae we obtain

$$
\begin{aligned}
p^{0}(P)\left(j^{1} \theta\right)= & \left(\left(\theta_{i}-\frac{\partial \theta_{0}}{\partial y^{i}}\right) \delta x^{0} \wedge \delta y^{i}+\frac{1}{2}\left(\frac{\partial \theta_{i}}{\partial y^{j}}-\frac{\partial \theta_{j}}{\partial y^{i}}\right) \delta x^{j} \wedge \delta x^{i},\right. \\
& \left.\left(\frac{\partial \theta_{i}}{\partial t}-\theta_{j} N_{i}^{j}-\frac{\delta \theta_{0}}{\delta x^{i}}\right) \delta x^{0} \wedge \delta x^{i}+\frac{1}{2}\left(\frac{\delta \theta_{i}}{\delta x^{j}}-\frac{\delta \theta_{j}}{\delta x^{i}}\right) \delta x^{j} \wedge \delta x^{i}\right) .
\end{aligned}
$$


The symbol of $P$ is the vector bundle morphism $\sigma^{1}(P): T^{*} \otimes T_{v}^{*} \rightarrow \Lambda^{2} T_{v}^{*} \oplus \Lambda^{2} T_{v}^{*}$ defined by the first-order terms of $p^{0}(P)$. More exactly, $\sigma^{1}(P)=p^{0}(P) \circ \epsilon$.

For $A \in T^{*} \otimes T_{v}^{*}, A=A_{\alpha \beta} \delta x^{\alpha} \otimes \delta x^{\beta}+A_{\underline{i} \beta} \delta y^{i} \otimes \delta x^{\beta}$, we compute

$$
\begin{aligned}
& \sigma^{1}\left(d_{J}\right) A=-A_{\underline{i} 0} \delta x^{0} \wedge \delta x^{i}+\frac{1}{2}\left(A_{\underline{j} i}-A_{\underline{j} j}\right) \delta x^{j} \wedge \delta x^{i}, \\
& \sigma^{1}\left(d_{h}\right) A=\left(A_{0 i}-A_{i 0}\right) \delta x^{0} \wedge \delta x^{i}+\frac{1}{2}\left(A_{j i}-A_{i j}\right) \delta x^{j} \wedge \delta x^{i}
\end{aligned}
$$

and hence

$$
\begin{aligned}
& \sigma^{1}(P) A=\left(\tau_{J} A, \tau_{h} A\right), \quad\left(\tau_{J} A\right)(X, Y)=A(J X, Y)-A(J Y, X), \\
& \left(\tau_{h} A\right)(X, Y)=A(h X, Y)-A(h Y, X),
\end{aligned}
$$

for $X, Y \in \mathfrak{X}\left(J^{1} \pi\right)$. In the above formulae $\tau_{J}, \tau_{L}$ are alternating operators [7].

Remark 3. The alternating operators are defined in general as follows. For $K \in \Psi^{k}\left(J^{1} \pi\right)$, a vector-valued $k$-form, we consider $\tau_{K}: \Psi^{1}\left(J^{1} \pi\right) \otimes \Psi^{l}\left(J^{1} \pi\right) \rightarrow \Psi^{l+k}\left(J^{1} \pi\right)$,

$$
\left(\tau_{K} B\right)\left(X_{1}, \ldots, X_{l+k}\right)=\frac{1}{l ! k !} \sum_{\sigma \in S_{l+k}} \varepsilon(\sigma) B\left(K\left(X_{\sigma(1)}, \ldots, X_{\sigma(k)}\right), X_{\sigma(k+1)}, \ldots, X_{\sigma(k+l)}\right),
$$

where $X_{1}, \ldots, X_{l+k} \in \mathfrak{X}\left(J^{1} \pi\right)$ and $S_{l+k}$ is the permutation group of $\{1, \ldots, l+k\}$. The restriction of $\tau_{K}$ to $\Psi^{l+1}\left(J^{1} \pi\right)$, is a derivation of degree $(k-1)$ and it coincides with the inner product $i_{K}$.

The first-order prolongation of the symbol of $P$ is the vector bundle morphism $\sigma^{2}(P): S^{2} T^{*} \otimes$ $T_{v}^{*} \rightarrow T^{*} \otimes\left(\Lambda^{2} T_{v}^{*} \oplus \Lambda^{2} T_{v}^{*}\right)$ that verifies

$$
i_{X}\left(\sigma^{2}(P) B\right)=\sigma^{1}(P)\left(i_{X} B\right), \quad \forall B \in S^{2} T^{*} \otimes T_{v}^{*}, \quad \forall X \in \mathfrak{X}\left(J^{1} \pi\right) .
$$

Therefore

$$
\begin{aligned}
& \sigma^{2}(P) B=\left(\sigma^{2}\left(d_{J}\right) B, \sigma^{2}\left(d_{h}\right) B\right), \\
& \left(\sigma^{2}\left(d_{J}\right) B\right)(X, Y, Z)=B(X, J Y, Z)-B(X, J Z, Y), \\
& \left(\sigma^{2}\left(d_{h}\right) B\right)(X, Y, Z)=B(X, h Y, Z)-B(X, h Z, Y) .
\end{aligned}
$$

In local coordinates we obtain the following formulae.

If $B \in S^{2} T^{*} \otimes T_{v}^{*}$, then it has the local decomposition

$$
\begin{aligned}
B= & B_{\alpha \beta \gamma} \delta x^{\alpha} \otimes \delta x^{\beta} \otimes \delta x^{\gamma}+B_{\underline{i} \alpha \beta} \delta y^{i} \otimes \delta x^{\alpha} \otimes \delta x^{\beta} \\
& +B_{\alpha \underline{i} \beta} \delta x^{\alpha} \otimes \delta y^{i} \otimes \delta x^{\beta}+B_{i \underline{j} \alpha} \delta y^{i} \otimes \delta y^{j} \otimes \delta x^{\alpha},
\end{aligned}
$$

with

$$
B_{\alpha \beta \gamma}=B_{\beta \alpha \gamma}, \quad B_{\underline{i j \alpha}}=B_{\underline{i j \alpha}}, \quad B_{\underline{i} 0 \alpha}=B_{0 \underline{i \alpha}}, \quad B_{\underline{i j \alpha}}=B_{\underline{j i \alpha}} .
$$

The first-order prolongation of the symbol of $P$ is given by

$$
\begin{aligned}
\sigma^{2}\left(d_{J}\right) B= & B_{\alpha \underline{i} 0} \delta x^{\alpha} \otimes \delta x^{i} \wedge \delta x^{0}+B_{\underline{i j} 0} \delta y^{i} \otimes \delta x^{j} \wedge \delta x^{0} \\
& +\frac{1}{2}\left(B_{\alpha \underline{i j}}-B_{\alpha \underline{j i}}\right) \delta x^{\alpha} \otimes \delta x^{i} \wedge \delta x^{j}+\frac{1}{2}\left(B_{\underline{i j} k}-B_{\underline{i k j} j}\right) \delta y^{i} \otimes \delta x^{j} \wedge \delta x^{k}, \\
\sigma^{2}\left(d_{h}\right) B= & \frac{1}{2}\left(B_{\alpha \beta \gamma}-B_{\alpha \gamma \beta}\right) \delta x^{\alpha} \otimes \delta x^{\beta} \wedge \delta x^{\gamma}+\frac{1}{2}\left(B_{\underline{i} \alpha \beta}-B_{\underline{i} \beta \alpha}\right) \delta y^{i} \otimes \delta x^{\alpha} \wedge \delta x^{\beta} .
\end{aligned}
$$


For each $u \in J^{1} \pi$, we consider

$$
\begin{aligned}
& g_{u}^{k}(P)=\operatorname{Ker} \sigma_{u}^{k}(P), \quad k \in\{1,2\}, \\
& g_{u}^{1}(P)_{e_{1} \ldots e_{j}}=\left\{A \in g_{u}^{1}(P) \mid i_{e_{1}} A=\cdots=i_{e_{j}} A=0\right\}, \quad j \in\{1, \ldots, n\},
\end{aligned}
$$

where $\left\{e_{1}, \ldots, e_{n}\right\}$ is a basis of $T_{u}\left(J^{1} \pi\right)$. Such a basis is called quasi-regular if it satisfies

$$
\operatorname{dim} g_{u}^{2}(P)=\operatorname{dim} g_{u}^{1}(P)+\sum_{j=1}^{n} \operatorname{dim} g_{u}^{1}(P)_{e_{1} \ldots e_{j}} .
$$

Definition 4. The symbol $\sigma^{1}(\mathcal{P})$ is called involutive at $u$ in $J^{1} \pi$ if there exists a quasi-regular basis of $T_{u} J^{1} \pi$.

A first-order jet $j_{u}^{1} \theta \in J^{1} T_{v}^{*}$ is a first-order formal solution of $P$ at $u$ in $J^{1} \pi$ if $p^{0}(P)(\theta)_{u}=0$.

For $l \geq 1$, a $(1+l)$ th order jet $j_{u}^{1+l} \theta \in J_{u}^{1+l} T_{v}^{*}$ is $a(1+l)$ th order formal solution of $P$ at $u$ in $J^{1} \pi$ if $p^{l}(P)(\theta)_{u}=0$.

For any $l \geq 0$, consider $R_{u}^{1+l}(P)=\operatorname{ker} p_{u}^{l}(P)$ the space of $(1+l)$ th order formal solutions of $P$ at $u$. We denote also $\bar{\pi}_{l, u}: R_{u}^{1+l}(P) \rightarrow R_{u}^{l}(P)$ the restriction of $\pi_{l, u}: J_{u}^{1+l}\left(T_{v}^{*}\right) \rightarrow J_{u}^{l}\left(T_{v}^{*}\right)$ to $R_{u}^{1+l}(P)$.

Definition 5. The partial differential operator $P$ is called formally integrable at $u$ in $J^{1} \pi$ if $R^{1+l}(P)=\bigcup_{u \in J^{1} \pi} R_{u}^{1+l}(P)$ is a vector bundle over $J^{1} \pi$, for all $l \geq 0$, and the map $\bar{\pi}_{l, u}$ : $R_{u}^{1+l}(P) \rightarrow R_{u}^{l}(P)$ is onto for all $l \geq 1$.

The fibred submanifold $R^{1}(P)$ of $\pi_{0}: J_{u}^{1}\left(T_{v}^{*}\right) \rightarrow J^{1} \pi$ is called the partial differential equation corresponding to the first-order PDO $P$. A solution of the operator $P$ on an open set $U \subset J^{1} \pi$ is a section $\theta \in \Lambda_{v}^{1}$ defined on $U$ such that $P \theta=0 \Leftrightarrow p^{0}(P)\left(j_{u}^{1} \theta\right)=0, \forall u \in U$.

The Cartan-Kähler theorem [23] takes the following form for the particular case of first-order PDO.

Theorem 2. Let $P$ be a first-order linear partial differential operator with $g^{2}(P)$ a vector bundle over $R^{1}(P)$. If $\bar{\pi}_{1}: R^{2}(P) \rightarrow R^{1}(P)$ is onto and the symbol $\sigma^{1}(P)$ is involutive, then $P$ is formally integrable.

\subsection{The involutivity of the symbol of $P$}

In this subsection we prove that the operator $P$ satisfies one of the two sufficient conditions for formal integrability, provided by Cartan-Kähler theorem: the involutivity of the symbol $\sigma^{1}(P)$.

Theorem 3. The symbol $\sigma^{1}(P)$ of the PDO $P=\left(d_{J}, d_{h}\right)$ is involutive.

Proof. First we determine $g^{1}(P)=\left\{A \in T^{*} \otimes T_{v}^{*} \mid \sigma^{1}(P) A=0\right\}$, and compute the dimension of its fibers. We obtain

$$
g_{u}^{1}(P)=\left\{A=A_{\alpha \beta} \delta x^{\alpha} \otimes \delta x^{\beta}+A_{\underline{i} \beta} \delta y^{i} \otimes \delta x^{\beta} \mid A_{\underline{i} 0}=0, A_{\underline{j} i}=A_{\underline{i} j}, A_{\alpha \beta}=A_{\beta \alpha}\right\} .
$$

From $A_{\underline{i} 0}=0$ and $A_{j i}=A_{\underline{i j}}$ it results that $A_{\underline{i j}}$ contribute with $n(n+1) / 2$ components to the dimension of $g_{u}^{1}(P)$, and from $A_{\alpha \beta}=A_{\beta \alpha}$ it follows that $A_{\alpha \beta}$ contribute with $(n+1)(n+2) / 2$ components to the dimension of $g_{u}^{1}(P)$. So

$$
\operatorname{dim} g_{u}^{1}(P)=\frac{n(n+1)}{2}+\frac{(n+1)(n+2)}{2}=(n+1)^{2} .
$$

Next we determine $g^{2}(P)=\left\{B \in S^{2} T^{*} \otimes T_{v}^{*} \mid \sigma^{2}(P) B=0\right\}$. 
If $B \in S^{2} T^{*} \otimes T_{v}^{*}$ has the local components (15), then $B \in g^{2}(P)$ if and only if the following relations are satisfied:

$$
\begin{aligned}
& B_{\alpha \underline{i} 0}=0, \quad B_{i j 0}=0, \quad B_{\alpha \underline{i j}}=B_{\alpha \underline{j} i}, \\
& B_{\underline{i j k}}=B_{\underline{i k j}}, \quad B_{\alpha \beta \gamma}=B_{\alpha \gamma \beta}, \quad B_{\underline{i} \alpha \beta}=B_{\underline{i} \beta \alpha} .
\end{aligned}
$$

From the relations (16) and (17) it results that $B \in g^{2}(P)$ if and only if its local components $B_{\alpha \beta \gamma}, B_{i j k}, B_{i j k}, B_{i j k}$ are totally symmetric and the rest are vanishing. Therefore $B_{\alpha \beta \gamma}$ contribute with $(n+1)(n+2)(n+3) / 6$ components to the dimension of $g_{u}^{2}(P)$, and $B_{\underline{i j k}}, B_{i j k}$ with $n(n+1)(n+2) / 6$ components each of them. It results

$$
\operatorname{dim} g_{u}^{2}(P)=\frac{(n+1)(n+2)(n+3)}{6}+2 \frac{n(n+1)(n+2)}{6}=\frac{(n+1)^{2}(n+2)}{2} .
$$

Consider

$$
\mathcal{B}=\left\{h_{0}=S, h_{1}, \ldots, h_{n}, v_{1}=J h_{1}, \ldots, v_{n}=J h_{n}\right\}
$$

a basis in $T_{u} J^{1} \pi$ with $h_{0}=S, h_{1}, \ldots, h_{n}$ horizontal vector fields. For any $A \in g^{1}(P)$, we denote

$$
A\left(h_{\alpha}, h_{\beta}\right)=a_{\alpha \beta}, \quad A\left(v_{i}, h_{\alpha}\right)=b_{\underline{i} \alpha} .
$$

Because $A$ is semi-basic in the second argument it follows that these are the only components of $A$.

Since $A \in \operatorname{Ker} \sigma^{1}\left(d_{J}\right)$ it follows that $A_{\underline{i} 0}=0, A_{\underline{i j}}=A_{\underline{j} i} \Rightarrow b_{\underline{i} 0}=0, b_{\underline{i} j}=b_{\underline{j} i}$. Because $A \in \operatorname{Ker} \sigma^{1}\left(d_{h}\right)$ it results that $A_{0 i}=A_{i 0}, A_{i j}=A_{j i}$ and hence $a_{0 i}=a_{i 0}, a_{i j}=a_{j i}$.

Consider $j \in\{1, \ldots, n\}$ arbitrarily fixed and

$$
\begin{aligned}
\tilde{\mathcal{B}}=\left\{e_{0}\right. & =S+h_{j}+v_{n}, e_{1}=h_{1}, e_{2}=h_{2}+v_{1}, \ldots, \\
& \left.e_{i}=h_{i}+v_{i-1}, \ldots, e_{n}=h_{n}+v_{n-1}, v_{1}, \ldots, v_{n}\right\}
\end{aligned}
$$

a new basis in $T_{u} J^{1} \pi$. If we denote

$$
A\left(e_{\alpha}, e_{\beta}\right)=\tilde{a}_{\alpha \beta}, \quad A\left(v_{i}, e_{\alpha}\right)=\tilde{b}_{\underline{i} \alpha},
$$

a simple computation and the fact that $A$ is semi-basic in the second argument determine

$$
\begin{aligned}
& \tilde{a}_{00}=a_{00}+2 a_{0 j}+a_{j j}+b_{\underline{n j}}, \\
& \tilde{a}_{i k}=a_{i k}+b_{\underline{i-1, k}} \neq \tilde{a}_{k i}=a_{k i}+b_{\underline{k-1, i}}, \\
& \tilde{a}_{i 0}=a_{i 0}+a_{i j}+b_{\underline{i-1}, j} \neq \tilde{a}_{0 i}=a_{0 i}+a_{j i}+b_{\underline{n} i}, \\
& \tilde{b}_{\underline{i} k}=b_{\underline{i} k}=\tilde{b}_{\underline{k} i}, \quad \tilde{b}_{\underline{i} 0}=b_{\underline{i j}} .
\end{aligned}
$$

It can be seen that all the independent components of $A$ in the basis $\mathcal{B}$ can be obtained from the components of $A$ in the basis $\tilde{\mathcal{B}}$, and hence we can use the later for determining the dimensions of $\left(g_{u}^{1}\right)_{e_{0} e_{1} \ldots e_{k}}$.

If $A \in\left(g_{u}^{1}\right)_{e_{0}}$ it results $\tilde{a}_{0 \alpha}=0$, so using this new basis we impose $n+1$ supplementary independent restrictions. It follows that $\operatorname{dim}\left(g_{u}^{1}\right)_{e_{0}}=(n+1)^{2}-(n+1)=(n+1) n$.

If $A \in\left(g_{u}^{1}\right)_{e_{0} e_{1}}$ it results that together with the previous restrictions we impose also $\tilde{a}_{1 \alpha}=0$, so another independent $n+1$ restrictions. Hence $\operatorname{dim}\left(g_{u}^{1}\right)_{e_{0} e_{1}}=(n+1) n-(n+1)=(n+1)(n-1)$.

In general $\operatorname{dim}\left(g_{u}^{1}\right)_{e_{0} e_{1} \ldots e_{k}}=(n+1)(n-k), \forall k \in\{1, \ldots, n\}$. Hence $\operatorname{dim}\left(g_{u}^{1}\right)_{e_{0} e_{1} \ldots e_{n}}=0 \Rightarrow$ $\operatorname{dim}\left(g_{u}^{1}\right)_{e_{0} \ldots e_{n} v_{1} \ldots v_{k}}=0, \forall k \in\{1, \ldots, n\}$,

$$
\begin{aligned}
\operatorname{dim}\left(g_{u}^{1}\right)+\sum_{k=0}^{n} \operatorname{dim}\left(g_{u}^{1}\right)_{e_{0} e_{1} \ldots e_{k}} & =(n+1)^{2}+(n+1)[n+n-1+\cdots+1] \\
& =\frac{(n+1)^{2}(n+2)}{2}=\operatorname{dim} g_{u}^{2}(P) .
\end{aligned}
$$

We proved that $\tilde{B}$ is a a quasi-regular basis, hence the symbol $\sigma^{1}(P)$ is involutive. 


\subsection{First obstruction to the inverse problem}

In this subsection we determine necessary and sufficient conditions for $\bar{\pi}_{1}$ to be onto. We will obtain only one obstruction for the integrability of the operator $P$. The obstruction is due to the curvature tensor of the nonlinear connection induced by the semispray.

Theorem 4. A first-order formal solution $\theta \in \Lambda_{v}^{1}$ of the system $d_{J} \theta=0, d_{h} \theta=0$ can be lifted into a second-order solution, which means that $\bar{\pi}_{1}: R^{2}(P) \rightarrow R^{1}(P)$ is onto, if and only if

$$
d_{R} \theta=0,
$$

where $R$ is the curvature tensor (4).

Proof. We use a known result from [23, Proposition 1.1].

If $\mathcal{K}$ is the cokernel of $\sigma^{2}(P)$,

$$
\mathcal{K}=\frac{T^{*} \otimes\left(\Lambda^{2} T_{v}^{*} \oplus \Lambda^{2} T_{v}^{*}\right)}{\operatorname{Im} \sigma^{2}(P)}
$$

there exists a morphism $\varphi: R^{1}(P) \rightarrow \mathcal{K}$ such that the sequence

$$
R^{2}(P) \stackrel{\bar{\pi}_{1}}{\longrightarrow} R^{1}(P) \stackrel{\varphi}{\longrightarrow} \mathcal{K}
$$

is exact. In particular $\bar{\pi}_{1}$ is onto if and only if $\varphi=0$.

After defining $\varphi$, we will prove that for $\theta \in \Lambda_{v}^{1}$, with $j_{u}^{1} \theta \in R_{u}^{1}(P)$, a first-order formal solution of $P$ at $u \in J^{1} \pi$, we have that $\varphi_{u} \theta=0$ if and only if $\left(d_{R} \theta\right)_{u}=0$.

The construction of the morphism $\varphi$ is represented in the next diagram by dashed arrows. We denote $F=\Lambda^{2} T_{v}^{*} \oplus \Lambda^{2} T_{v}^{*}$.

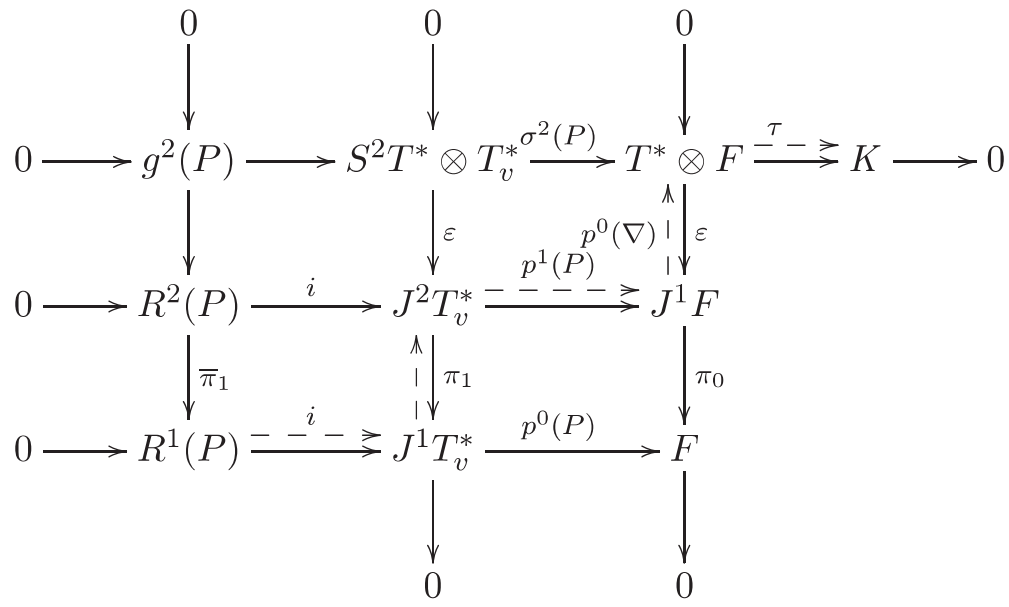

Remark that $\operatorname{dim} T^{*}=2 n+1, \operatorname{dim} T_{v}^{*}=n+1, \operatorname{dim} \Lambda^{2} T_{v}^{*}=\frac{(n+1) n}{2}, \operatorname{dim} \mathcal{S}^{2} T^{*}=\frac{(2 n+1)(2 n+2)}{2}$, $\operatorname{dim} T^{*} \otimes\left(\Lambda^{2} T_{v}^{*} \oplus \Lambda^{2} T_{v}^{*}\right)=(2 n+1) n(n+1)$. Therefore

$$
\begin{aligned}
\operatorname{dim} \mathcal{K} & =\operatorname{dim}\left[T^{*} \otimes\left(\Lambda^{2} T_{v}^{*} \oplus \Lambda^{2} T_{v}^{*}\right)\right]-\operatorname{dim}\left(\operatorname{Im} \sigma^{2}(P)\right) \\
& =\operatorname{dim}\left[T^{*} \otimes\left(\Lambda^{2} T_{v}^{*} \oplus \Lambda^{2} T_{v}^{*}\right)\right]-\left[\operatorname{dim}\left(\mathcal{S}^{2} T^{*} \otimes T_{v}^{*}\right)-\operatorname{dim}\left(\operatorname{ker} \sigma^{2}(P)\right)\right] \\
& =\frac{(n-1) n(n+1)}{2}=3\left(\begin{array}{c}
n+1 \\
3
\end{array}\right) .
\end{aligned}
$$

It results from this that

$$
\mathcal{K} \simeq \oplus^{(3)} \Lambda^{3} T_{v}^{*}
$$


Next we define $\tau: T^{*} \otimes\left(\Lambda^{2} T_{v}^{*} \oplus \Lambda^{2} T_{v}^{*}\right) \rightarrow \oplus^{(3)} \Lambda^{3} T_{v}^{*}$ such as the next sequence is exact:

$$
0 \rightarrow g^{2}(P) \stackrel{i}{\longrightarrow} \mathcal{S}^{2} T^{*} \otimes T_{v}^{*} \stackrel{\sigma^{2}(P)}{\longrightarrow} T^{*} \otimes\left(\Lambda^{2} T_{v}^{*} \oplus \Lambda^{2} T_{v}^{*}\right) \stackrel{\tau}{\longrightarrow} \oplus^{(3)} \Lambda^{3} T_{v}^{*} \rightarrow 0 .
$$

For $B_{1}, B_{2} \in T^{*} \otimes \Lambda^{2} T_{v}^{*}$, we define $\tau\left(B_{1}, B_{2}\right)=\left(\tau_{1}\left(B_{1}, B_{2}\right), \tau_{2}\left(B_{1}, B_{2}\right), \tau_{3}\left(B_{1}, B_{2}\right)\right)$, where $\tau_{i}: T^{*} \otimes\left(\Lambda^{2} T_{v}^{*} \oplus \Lambda^{2} T_{v}^{*}\right) \rightarrow \Lambda^{3} T_{v}^{*}, i \in\{1,2,3\}$, are given by

$$
\tau_{1}\left(B_{1}, B_{2}\right)=\tau_{J} B_{1}, \quad \tau_{2}\left(B_{1}, B_{2}\right)=\tau_{h} B_{2}, \quad \tau_{3}\left(B_{1}, B_{2}\right)=\tau_{h} B_{1}+\tau_{J} B_{2} .
$$

Using the definition (14) of the alternating operators $\tau_{J}, \tau_{h}$, we prove that $\tau \circ \sigma^{2}(P)=0$.

Indeed, using that any $B \in \mathcal{S}^{2} T^{*} \otimes T_{v}^{*}$ is symmetric in the first two arguments, it follows that $\left(\tau \circ \sigma^{2}(P)\right)(B)=\tau\left(\sigma^{2}\left(d_{J}\right) B, \sigma^{2}\left(d_{h}\right) B\right)=\left(\tau_{J} \sigma^{2}\left(d_{J}\right) B, \tau_{h} \sigma^{2}\left(d_{h}\right) B, \tau_{h} \sigma^{2}\left(d_{J}\right) B+\tau_{J} \sigma^{2}\left(d_{h}\right) B\right)=$ $0, \forall B \in \mathcal{S}^{2} T^{*} \otimes T_{v}^{*}$. For example,

$$
\begin{aligned}
\tau_{J}\left(\sigma^{2}\left(d_{J}\right) B\right)(X, Y, Z) \\
=\left[\sigma^{2}\left(d_{J}\right) B(J X, Y, Z)-\sigma^{2}\left(d_{J}\right) B(J Y, X, Z)+\sigma^{2}\left(d_{J}\right) B(J Z, X, Y)\right] \\
=[B(J X, J Y, Z)-B(J X, J Z, Y)-B(J Y, J X, Z)+B(J Y, J Z, X) \\
+B(J Z, J X, Y)-B(J Z, J Y, X)]=0, \quad \forall X, Y, Z \in \mathfrak{X}\left(J^{1} \pi\right) .
\end{aligned}
$$

The relation $\tau \circ \sigma^{2}(P)=0$ implies that $\operatorname{Im}\left(\sigma^{2}(P)\right) \subseteq \operatorname{Ker} \tau$. Using that $\tau$ is onto $\left(\tau_{J}, \tau_{h}\right.$ are both onto) it results that $\operatorname{dim}\left[\operatorname{Im}\left(\sigma^{2}(P)\right)\right]=\operatorname{dim}(\operatorname{Ker} \tau)$ and hence $\operatorname{Im}\left(\sigma^{2}(P)\right)=\operatorname{Ker} \tau$ and the sequence (18) is exact.

The last step before defining $\varphi: R^{1}(P) \rightarrow \mathcal{K}$ is to consider a linear connection $\nabla$ on $J^{1} \pi$ such that $\nabla J=0$. It means that $\nabla$ preserve semi-basic forms and $\nabla$ can be considered as a connection in the fiber bundle $\Lambda^{2} T_{v}^{*} \oplus \Lambda^{2} T_{v}^{*} \rightarrow J^{1} \pi$. As a first-order PDO we can identify $\nabla$ with the bundle morphism $p^{0}(\nabla): J^{1}\left(\Lambda^{2} T_{v}^{*} \oplus \Lambda^{2} T_{v}^{*}\right) \rightarrow T^{*} \otimes\left(\Lambda^{2} T_{v}^{*} \oplus \Lambda^{2} T_{v}^{*}\right)$.

We will also use two derivations of degree 1 introduced in [7], defined by $\mathcal{D}_{J}=\tau_{J} \nabla, \mathcal{D}_{h}=\tau_{h} \nabla$. Both derivations $\mathcal{D}_{J}, \mathcal{D}_{h}$ preserve semi-basic forms and $d_{J}-\mathcal{D}_{J}, d_{h}-\mathcal{D}_{h}$ are algebraic derivations. It means that if $\omega \in \Lambda^{k}\left(J^{1} \pi\right)$ vanishes at some point $u \in J^{1} \pi$, then $\left(\mathcal{D}_{J} \omega\right)_{u}=\left(d_{J} \omega\right)_{u}$ and $\left(\mathcal{D}_{h} \omega\right)_{u}=\left(d_{h} \omega\right)_{u}[7$, Lemma 2.1].

Now we are able to define $\varphi: R^{1}(P) \rightarrow \mathcal{K}$ such that the sequence

$$
R^{2}(P) \stackrel{\bar{\pi}_{1}}{\longrightarrow} R^{1}(P) \stackrel{\varphi}{\longrightarrow} \mathcal{K}
$$

is exact.

Let $\theta \in \Lambda_{v}^{1}$ such that $j_{u}^{1} \theta \in R_{u}^{1}(P) \subset J_{u}^{1} T_{v}^{*}$ is a first-order formal solution of $P$ at $u \in J^{1} \pi$, which means that $\left(d_{J} \theta\right)_{u}=\left(d_{h} \theta\right)_{u}=0$.

Consider

$$
\varphi_{u} \theta=\tau_{u} \nabla P \theta=\tau_{u}\left(\nabla d_{J} \theta, \nabla d_{h} \theta\right) .
$$

Using the fact that $d_{J}-\tau_{J} \nabla$ and $d_{h}-\tau_{h} \nabla$ are algebraic derivations and $\left(d_{J} \theta\right)_{u}=\left(d_{h} \theta\right)_{u}=0$ it results $d_{J}\left(d_{J} \theta\right)_{u}=\tau_{J} \nabla\left(d_{J} \theta\right)_{u}$ and $d_{h}\left(d_{h} \theta\right)_{u}=\tau_{h} \nabla\left(d_{h} \theta\right)_{u}$.

We will compute the three components of the map $\varphi$. It follows that

$$
\begin{aligned}
\tau_{1}(\nabla P \theta)_{u} & =\tau_{1}\left(\nabla d_{J} \theta, \nabla d_{h} \theta\right)_{u}=\tau_{J}\left(\nabla d_{J} \theta\right)_{u} \\
& =\left(d_{J}^{2} \theta\right)_{u}=\frac{1}{2}\left(d_{[J, J]} \theta\right)_{u}=-\left(d_{J \wedge d t} \theta\right)_{u}=-\left(d_{J} \theta\right)_{u} \wedge d t=0, \\
\tau_{2}(\nabla P \theta)_{u} & =\tau_{2}\left(\nabla d_{J} \theta, \nabla d_{h} \theta\right)_{u}=\tau_{h}\left(\nabla d_{h} \theta\right)_{u}=\left(d_{h}^{2} \theta\right)_{u}=\frac{1}{2}\left(d_{[h, h]} \theta\right)_{u}=\left(d_{R} \theta\right)_{u},
\end{aligned}
$$


where $R$ is given by (4),

$$
\begin{aligned}
\tau_{3}(\nabla P \theta)_{u} & =\tau_{3}\left(\nabla d_{J} \theta, \nabla d_{h} \theta\right)_{u}=\tau_{h}\left(\nabla d_{J} \theta\right)_{u}+\tau_{J}\left(\nabla d_{h} \theta\right)_{u} \\
& =\left(d_{h} d_{J} \theta\right)_{u}+\left(d_{J} d_{h} \theta\right)_{u}=\left(d_{[h, J]} \theta\right)_{u}=0 .
\end{aligned}
$$

Hence $\varphi=0$ if and only if $d_{R} \theta=0$.

Remark 4. Locally, $d_{R} \theta$ has the following form:

$$
\begin{aligned}
& R=\underbrace{\frac{1}{2} R_{i j}^{k} \frac{\partial}{\partial y^{k}} \otimes \delta x^{i} \wedge \delta x^{j}}_{\widetilde{R}=\frac{1}{3}[J, \Phi]}-\Phi \wedge d t \Rightarrow d_{R} \theta=d_{\widetilde{R}} \theta-d_{\Phi} \theta \wedge d t, \\
& d_{\Phi} \theta=R_{i}^{j}\left(\theta_{j}-\frac{\partial \theta_{0}}{\partial y^{j}}\right) d t \wedge \delta x^{i}+\frac{1}{2 !}\left(\frac{\partial \theta_{j}}{\partial y^{k}} R_{i}^{k}-\frac{\partial \theta_{i}}{\partial y^{k}} R_{j}^{k}\right) \delta x^{j} \wedge \delta x^{i} \Rightarrow \\
& d_{R} \theta=\frac{1}{3 !}\left(a_{i l} R_{j k}^{l}+a_{j l} R_{k i}^{l}+a_{k l} R_{i j}^{l}\right) \delta x^{i} \wedge \delta x^{j} \wedge \delta x^{k}+\frac{1}{2 !}\left(a_{j k} R_{i}^{k}-a_{i k} R_{j}^{k}\right) d t \wedge \delta x^{i} \wedge \delta x^{j},
\end{aligned}
$$

where we denoted $a_{i j}=\frac{\partial \theta_{i}}{\partial y^{j}}$.

Hence $d_{R} \theta=0$ if and only if $a_{i l} R_{j k}^{l}+a_{j l} R_{k i}^{l}+a_{k l} R_{i j}^{l}=0$ and $a_{j k} R_{i}^{k}-a_{i k} R_{j}^{k}=0$. The first identity represents the algebraic Bianchi identity for the curvatures of the nonlinear connection. The second identity is one of the classical Helmholtz condition for the multiplier matrix $a_{i j}$. These obstructions appear also in [2].

It can be seen that for $n=2$ the formula of $d_{R} \theta$ becomes

$$
d_{R} \theta=\frac{1}{2 !}\left(a_{j k} R_{i}^{k}-a_{i k} R_{j}^{k}\right) d t \wedge \delta x^{i} \wedge \delta x^{j}=-d_{\Phi} \theta \wedge d t
$$

Therefore, for $n=2$, the obstruction is equivalent with $d_{\Phi} \theta \wedge d t=0$.

\subsection{Classes of Lagrangian time-dependent SODE}

We present now some classes of semisprays for which the obstruction in Theorem 4 is automatically satisfied. Therefore the PDO $P$ is formally integrable, and hence these semisprays will be Lagrangians SODEs. These classes of semisprays are:

- flat semisprays, $R=0 \Leftrightarrow \Phi=0$;

- arbitrary semisprays on 2-dimensional manifolds;

- isotropic semisprays, $\Phi=\lambda J$, for $\lambda$ a smooth function on $J^{1} \pi$.

All these classes of semisprays were already studied in the articles cited in the introduction.

In the flat case, the obstruction is automatically satisfied.

If $\operatorname{dim} M=1$ then for a semi-basic 1-form $\theta$ on $J^{1} \pi, d_{R} \theta$ is a semi-basic 3 -form on $J^{1} \pi$. Because $\operatorname{dim} \Lambda^{3}\left(T_{v}^{*}\right)=(n+1) n(n-1) / 6$ and it is zero if $n=1, d_{R} \theta$ will necessarily vanish.

We consider now the last case, of isotropic semisprays.

Proposition 2. Any isotropic semispray is a Lagrangian second-order vector field.

Proof. Assume now that $S$ is an isotropic SODE and $\theta$ a semi-basic 1-form on $J^{1} \pi$ such that $\left(d_{J} \theta\right)_{u}=\left(d_{h} \theta\right)_{u}=0$, for some $u \in J^{1} \pi$,

$$
\left(d_{R} \theta\right)_{u}=d_{\alpha \wedge J} \theta=\alpha \wedge d_{J} \theta+(-1)^{2} d \alpha \wedge i_{J} \theta=0 .
$$

We used that $i_{J} \theta=0,\left(d_{J} \theta\right)_{u}=0$ and the formula [23]

$$
d_{\omega \wedge K} \pi=\omega \wedge d_{K} \pi+(-1)^{q+k} d \omega \wedge i_{K} \pi,
$$

for $\omega$ a $q$-form on $J^{1} \pi$ and $K$ a vector-valued $k$-form on $J^{1} \pi$.

Since $\left(d_{R} \theta\right)_{u}$ vanishes, $S$ is a Lagrangian semispray. 
Next we give some simple examples of Lagrangian semisprays, corresponding to the above general classes.

We start with the semispray expressed by the SODE

$$
\frac{d^{2} x^{1}}{d t^{2}}+f\left(t, \frac{d x^{2}}{d t}\right)=0, \quad \frac{d^{2} x^{2}}{d t^{2}}+g(t)=0
$$

with $f$ an arbitrary smooth function depending only on $t$ and $y^{2}=\frac{d x^{2}}{d t}$, and $g$ an arbitrary smooth function depending only on $t$. The only possible nonvanishing local component of the Jacobi endomorphism is

$$
R_{2}^{1}=-S\left(N_{2}^{1}\right)=-\frac{1}{2} \frac{\partial^{2} f}{\partial t \partial y^{2}}+g(t) \frac{\partial^{2} f}{\partial\left(y^{2}\right)^{2}}
$$

Hence, if $\frac{\partial^{2} f}{\partial t \partial y^{2}}=2 g(t) \frac{\partial^{2} f}{\partial\left(y^{2}\right)^{2}}$ the semispray is flat $(\Phi=0)$. This example is a generalization of the one given by Douglas [20, (8.14)].

If $\frac{\partial^{2} f}{\partial t \partial y^{2}} \neq 2 g(t) \frac{\partial^{2} f}{\partial\left(y^{2}\right)^{2}}$, then the semispray is isotropic

$$
\Phi=\left(\begin{array}{cc}
0 & -\frac{1}{2} \frac{\partial^{2} f}{\partial t \partial y^{2}}+g(t) \frac{\partial^{2} f}{\partial\left(y^{2}\right)^{2}} \\
0 & 0
\end{array}\right)=\left(-\frac{1}{2} \frac{\partial^{2} f}{\partial t \partial y^{2}}+g(t) \frac{\partial^{2} f}{\partial\left(y^{2}\right)^{2}}\right) J .
$$

Another example of isotropic (or flat) semispray is the one given by the SODE

$$
\frac{d^{2} x^{1}}{d t^{2}}+f\left(t, x^{2}\right)=0, \quad \frac{d^{2} x^{2}}{d t^{2}}+g(t)=0,
$$

with $f$ an arbitrary smooth function depending only on $t$ and $x^{2}$, and $g$ an arbitrary smooth function depending only on $t$. All the local coefficients of the associated nonlinear connection are vanishing. Evidently

$$
\Phi=\left(\begin{array}{cc}
0 & \frac{\partial f}{\partial x^{2}} \\
0 & 0
\end{array}\right)=\left(\frac{\partial f}{\partial x^{2}}\right) J
$$

This example was treated in $[2,(6.1)]$ and $[20,(15.4)]$ for $f\left(x^{2}\right)=-x^{2}$ and $g=0$. The first paper also presents all the Lagrangians corresponding to the given SODE.

Consider also the semispray given by the SODE

$$
\frac{d^{2} x^{1}}{d t^{2}}+2 \frac{d x^{2}}{d t}=0, \quad \frac{d^{2} x^{2}}{d t^{2}}-\left(\frac{d x^{2}}{d t}\right)^{2}=0 .
$$

Evidently

$$
\Phi=\left(\begin{array}{cc}
0 & y^{2} \\
0 & 0
\end{array}\right)
$$

hence the semispray is isotropic.

\section{Acknowledgements}

The author express his thanks to Ioan Bucataru for the many interesting discussions about the paper. 


\section{References}

[1] Aldridge J.E., Prince G.E., Sarlet W., Thompson G., An EDS approach to the inverse problem in the calculus of variations, J. Math. Phys. 47 (2006), 103508, 22 pages.

[2] Anderson I., Thompson G., The inverse problem of the calculus of variations for ordinary differential equations, Mem. Amer. Math. Soc. 98 (1992), no. 473, 110 pages.

[3] Antonelli P.L., Bucataru I., Volterra-Hamilton production models with discounting: general theory and worked examples, Nonlinear Anal. Real World Appl. 2 (2001), 337-356.

[4] Bryant R.L., Chern S.S., Gardner R.B., Goldschmidt H.L., Griffiths P.A., Exterior differential systems, Mathematical Sciences Research Institute Publications, Vol. 18, Springer-Verlag, New York, 1991.

[5] Bucataru I., Constantinescu O., Helmholtz conditions and symmetries for the time dependent case of the inverse problem of the calculus of variations, J. Geom. Phys. 60 (2010), 1710-1725, arXiv:0908.1631.

[6] Bucataru I., Dahl M.F., Semi-basic 1-forms and Helmholtz conditions for the inverse problem of the calculus of variations, J. Geom. Mech. 1 (2009), 159-180, arXiv:0903.1169.

[7] Bucataru I., Muzsnay Z., Projective metrizability and formal integrability, SIGMA 7 (2011), 114, 22 pages, arXiv:1105.2142.

[8] Cantrijn F., Cariñena J.F., Crampin M., Ibort L.A., Reduction of degenerate Lagrangian systems, J. Geom. Phys. 3 (1986), 353-400.

[9] Cantrijn F., Sarlet W., Vandecasteele A., Martínez E., Complete separability of time-dependent second-order ordinary differential equations, Acta Appl. Math. 42 (1996), 309-334.

[10] Cariñena J.F., Gràcia X., Marmo G., Martínez E., Muñoz-Lecanda M.C., Román-Roy N., Geometric Hamilton-Jacobi theory, Int. J. Geom. Methods Mod. Phys. 3 (2006), 1417-1458, math-ph/0604063.

[11] Cariñena J.F., Martínez E., Symmetry theory and Lagrangian inverse problem for time-dependent secondorder differential equations, J. Phys. A: Math. Gen. 22 (1989), 2659-2665.

[12] Cartan É., Les systèmes différentiels extérieurs et leurs applications géométriques, Actualités Sci. Ind., no. 994, Hermann et Cie., Paris, 1945.

[13] Crampin M., On the differential geometry of the Euler-Lagrange equations, and the inverse problem of Lagrangian dynamics, J. Phys. A: Math. Gen. 14 (1981), 2567-2575.

[14] Crampin M., Prince G.E., Sarlet W., Thompson G., The inverse problem of the calculus of variations: separable systems, Acta Appl. Math. 57 (1999), 239-254.

[15] Crampin M., Prince G.E., Thompson G., A geometrical version of the Helmholtz conditions in timedependent Lagrangian dynamics, J. Phys. A: Math. Gen. 17 (1984), 1437-1447.

[16] Crampin M., Sarlet W., Martínez E., Byrnes G.B., Prince G.E., Towards a geometrical understanding of Douglas' solution of the inverse problem of the calculus of variations, Inverse Problems 10 (1994), 245-260.

[17] Darboux G., Leçons sur la théorie générale des surfaces et les applications géométriques du calcul infinitésimal, Gauthier-Villars, Paris, 1894.

[18] Davis D.R., The inverse problem of the calculus of variations in a space of $(n+1)$ dimensions, Bull. Amer. Math. Soc. 35 (1929), 371-380.

[19] de Leon M., Rodrigues P.R., Dynamical connections and non-autonomous Lagrangian systems, Ann. Fac. Sci. Toulouse Math. (5) 9 (1988), 171-181.

[20] Douglas J., Solution of the inverse problem of the calculus of variations, Trans. Amer. Math. Soc. 50 (1941), $71-128$.

[21] Frölicher A., Nijenhuis A., Theory of vector-valued differential forms. I. Derivations of the graded ring of differential forms, Nederl. Akad. Wetensch. Proc. Ser. A 59 (1956), 338-359.

[22] Goldschmidt H., Integrability criteria for systems of nonlinear partial differential equations, J. Differential Geometry 1 (1967), 269-307.

[23] Grifone J., Muzsnay Z., Variational principles for second-order differential equations. Application of the Spencer theory to characterize variational sprays, World Scientific Publishing Co. Inc., River Edge, NJ, 2000.

[24] Klein J., Espaces variationnels et mécanique, Ann. Inst. Fourier (Grenoble) 12 (1962), 1-124.

[25] Kolář I., Michor P.W., Slovák J., Natural operations in differential geometry, Springer-Verlag, Berlin, 1993.

[26] Kosambi D., Systems of differential equations of the second order, Q. J. Math. 6 (1935), 1-12. 
[27] Krupková O., The geometry of ordinary variational equations, Lecture Notes in Mathematics, Vol. 1678, Springer-Verlag, Berlin, 1997.

[28] Krupková O., Prince G.E., Second order ordinary differential equations in jet bundles and the inverse problem of the calculus of variations, in Handbook of Global Analysis, Elsevier Sci. B.V., Amsterdam, 2008, 837-904.

[29] Massa E., Pagani E., Jet bundle geometry, dynamical connections, and the inverse problem of Lagrangian mechanics, Ann. Inst. H. Poincaré Phys. Théor. 61 (1994), 17-62.

[30] Prástaro A., (Co)bordism groups in PDEs, Acta Appl. Math. 59 (1999), 111-201.

[31] Prástaro A., Geometry of PDE's. II. Variational PDE's and integral bordism groups, J. Math. Anal. Appl. 321 (2006), 930-948.

[32] Riquier C., Les systèmes d'équations aux dérivées partielles, Gauthier-Villars, Paris, 1910.

[33] Santilli R.M., Foundations of theoretical mechanics. I. The inverse problem in Newtonian mechanics, Texts and Monographs in Physics, Springer-Verlag, New York - Heidelberg, 1978.

[34] Sarlet W., Symmetries, first integrals and the inverse problem of Lagrangian mechanics, J. Phys. A: Math. Gen. 14 (1981), 2227-2238.

[35] Sarlet W., The Helmholtz conditions revisited. A new approach to the inverse problem of Lagrangian dynamics, J. Phys. A: Math. Gen. 15 (1982), 1503-1517.

[36] Sarlet W., Crampin M., Martínez E., The integrability conditions in the inverse problem of the calculus of variations for second-order ordinary differential equations, Acta Appl. Math. 54 (1998), 233-273.

[37] Sarlet W., Thompson G., Prince G.E., The inverse problem of the calculus of variations: the use of geometrical calculus in Douglas's analysis, Trans. Amer. Math. Soc. 354 (2002), 2897-2919.

[38] Sarlet W., Vandecasteele A., Cantrijn F., Martínez E., Derivations of forms along a map: the framework for time-dependent second-order equations, Differential Geom. Appl. 5 (1995), 171-203.

[39] Saunders D.J., The geometry of jet bundles, London Mathematical Society Lecture Note Series, Vol. 142, Cambridge University Press, Cambridge, 1989.

[40] Spencer D.C., Overdetermined systems of linear partial differential equations, Bull. Amer. Math. Soc. 75 (1969), 179-239. 